\title{
Current Native Advertising Practices and Disclosures: An Abstract
}

\author{
Hillary A. Leonard, Christy Ashley, and Christine M. Kowalczyk
}

\begin{abstract}
Native advertising, or paid content that is assimilated with the visual design and function of nonpaid content on a publisher's site, is growing in popularity. The Interactive Advertising Bureau (IAB) asserts widespread advertiser and publisher agreement regarding clear and prominent disclosure that native ads are paid content. The disclosures are important because they help prevent scrutiny about whether native advertising is misleading or deceptive, which could trigger policy intervention. However, by definition, native ads, including their disclosures, are designed to match the experience provided by other content. So, it is not clear (a) if consumers know native ads are sponsored content or (b) whether consumers care about the source of the content. Thus, the initial research questions are How are advertisers using native advertising? How are they disclosing their sponsorship of the content? To begin to address this gap in our understanding of native advertising, the article begins with a report of current native advertising formats and practices. It then reviews 122 examples of native ads from identified native advertising leaders over a 31-day period on October 2014. The report summarizes ad format (video, content, picture), industries that used native ads, the amount of engagement with the content during the 31-day period, and, perhaps most importantly, how the sponsorship was disclosed in each case. Most native ads that were recognized were instream ads that used words (vs. video or pictures). However, videos had higher levels of engagement (as measured by social media). Videos also seemed more likely to use celebrities and emotional appeals. Technology companies were the dominant users of native ads during the time period. Substantial variation existed in words used, shading, colors, capitalization, contrast between text and background color, font size, and timing/placement of disclosures. The manuscript contributes to the conceptualization of native advertising, provides an overview of disclosures currently used, and provides directions for next steps and future inquiry by marketers, policy makers, and policy influencers.
\end{abstract}

\author{
H.A. Leonard $(\square) \bullet$ C. Ashley \\ University of Rhode Island, Kingston, RI, USA \\ e-mail: HLeonard@uri.edu; ChristyAshley@uri.edu \\ C.M. Kowalczyk \\ East Carolina University, Greenville, NC, USA \\ e-mail: KowalczykC@ecu.edu
}

